\title{
Cross-sectional study on community knowledge and perception on mental illness among residents of sub-zoba serejaka (Embaderho and Geshinashim villages), Eritrea
}

\begin{abstract}
Introduction: Mental illness is one of the increasing non-communicable diseases globally. The epidemicity of mental illness is mainly related to different structural and intermediary determinants of health inequities. Despite the rising of mental illnesses the knowledge and perception regarding mental illnesses is not known among different communities in Eritrea
\end{abstract}

Methods: A community based cross-sectional study among 141 selected households of Sub-Zoba Serejaka (Embaderho and Geshinashim villages) was conducted, from December 11 to $16,2017$.

Results: Out of the total $(n=141)$ study participants, $120(85.1 \%)$ were females. Mean age of the participants was 41.36 years $(\mathrm{SD} \pm 14.32)$. Majority $(85.8 \%)$ were farmers, Tigrigna ethnic group $(99.3 \%)$ and orthodox $(93.6 \%)$ by religion. Seventy three $(51.8 \%)$ of the study participants were below junior high school education level and house wives were $55.3 \%$. The study participants had either agreed or strongly agreed on the causes of mental illness that include: of substance misuse $(70.9 \%)$ head injury $(83.4 \%)$, and stress $(83 \%)$; whereas $70.9 \%$ noted that it is curable but equal number argued that they agree, disagree or not sure that mental illness can be caused or not by communicable or non-communicable diseases. They disagreed or strongly disagreed that mental illness is punishment from God $(64.5 \%)$ or caused by evil sprites (49.7\%); but equal number noted that either it can be genetically inherited or not. $85.1 \%$ agreed that mental illness is a curable disease and $68.8 \%$ were found to decide the best preferable site for treatment as the modern medical approach. The study results showed no association between sex, monthly income and preference for modern medical approach but a statistically significant association was found between preference for modern medical approach, age $(\mathrm{p}-$ value $=0.032)$ and the literacy $(\mathrm{p}-$ value $=0.000)$.

Discussion and conclusion: Majority of the study participants in the community of the study area were found to have good knowledge and perception on the causes, cure and preference of treatment approach of mental illness. Poor perception was observed among old aged and less educated individuals. Even though $85.1 \%$ of the study participants noted that it is curable but only $68.8 \%$ preferred to use modern medical approach. Therefore, the study results recommend an intensified health promotion activity to create awareness and clear understanding that mental illness is not caused by evil spirits, punishment from God or bewitched and to make them to understand that the preferred treatment approach is modern medical approach. This might be implemented by utilizing community health workers in an area with a shortage of mental health care staff.

Keywords: mental illnesses, knowledge, perception, community
Volume 7 Issue 3 - 2018

\author{
Elias Teages Adgoy,' Rezene Habtemariam² \\ IDepartment of Community Medicine and Primary Health \\ Care, Orotta School of Medicine and Dentistry, Eritrea \\ 2Department of Psychology, Orotta School of Medicine and \\ Dentistry, Eritrea
}

Correspondence: Elias Teages Adgoy, Department of Community Medicine and Primary Health Care, Orotta School of Medicine and Dentistry,Asmara, Eritrea, Email eliasteages@gmail.com

Received: May 04, 2018 | Published: May 22, 2018

\begin{abstract}
Abbreviations: AIDS, acquired immuno-deficiency syndromes; CDC, communicable diseases control; $\mathrm{CSDH}$, conceptual frame work for social determinants of health; HIV, human immuno-deficiency viruses; $\mathrm{MOH}$, ministry of health; STDs, sexually transmitted diseases; WHO, world health organization
\end{abstract}

\section{Introduction}

Non- communicable diseases are becoming epidemic with a need of timely public health interventions for enabling individuals to prevent the increase of these diseases that include mainly mental illness, hypertension, diabetes, injury and chronic respiratory diseases. Mental illness is usually related to different structural and intermediary determinants of health inequities that include: the socioeconomic and political context. The structural determinants of health inequities are: governance; macro-economic, social polices (labor market, housing, land) and public polices (education, health, social protection); cultural and social values. The structural determinants also include: socio-economic position, social class, gender, ethnicity, education, occupation and income. Whereas the 
intermediary determinants of health include: material circumstances (living and working condition, food availability, etc...); behavioral and biological and psychosocial factors that affect the health system and go round in impacting negatively an equity in health and well being. ${ }^{1}$ Some of the factors that influence negatively mental health services include: lack of trained mental health specialists; lack of prioritization and absence of clear mental health policy; poor health facilities and less equipped infrastructure; lack of evidence-based and culture associated assessment, care and treatment. ${ }^{2}$ Traditional healing has a long history in most low income developing countries and still it is widely practiced. In most countries with poor mental health care settings (scarcity of human and material resources) traditional healing practices of mental illnesses becomes dominant.

A study of India that was conducted for assessing Perceptions of Traditional Healing for Mental Illness, reported that drinking holy water and prayer as the most common treatment interventions ranking $3^{\text {rd }}$ and $4^{\text {th }}$ and it was practiced mainly by the traditional healers. The study also informed that $42.8 \%$ of the study participants responded that mental illness is curable. ${ }^{3}$ A study on Community Perception towards Mental Illness, that was conducted in Ethiopia (2016), reported poor perception towards mental illness as $37.3 \%$ and majority of the respondents replied that mental illness is either caused by supernatural powers (evil spirit), God's punishment or witchcraft. ${ }^{4}$ A study in a rural community of Kenya (2007), documented a response of $53 \%$ that mental illness is communicable and the remaining $47 \%$ mentioned that it is not communicable; it can be transmitted by physical contact $(18 \%)$ and genetic inheritance $(82 \%) .^{5}$ There is a study that reported peoples beliefs on the incurability of mental illness is mainly related to its causes that include; witchcraft, punishment from God due to wrong performance and the best treatment for such diseases spiritual mediations rather than medical approach. ${ }^{6}$ Therefore, despite the increased burden of mental health problem, little is known about communities' knowledge and perception towards mental health problems in Eritrea. Hence this cross-sectional study was conducted in two villages of Sub - Zoba Serejaka (Embadeho and Geshinashim) for assessing community knowledge and perception on mental illness that will help as baseline information.

\section{Objective of the study}

The objective of the study was to assess the knowledge and perception of the community towards the causes of mental illness and to identify the preferred place of treatment for mental illness in the community.

\section{Methodology}

\section{Study design and study period}

A community based cross-sectional study on the knowledge and perception of community and preferred place of treatment was conducted from December 11 to 16, 2017, at Embaderho and Geshinashim villages that are located at 12 and 28 kilometers to the west of the capital city, Asmara, Eritrea. Embaderho has one health center with an estimated number of 1,910 households and Geshinashim also has one clinic with an estimated number of 219 households.

\section{Sampling techniques}

Following purposive selection of two villages in the Sub - Zoba, households were selected by systematic random sampling. The first household was selected by simple random sampling. From each selected household, the head of the household (wife or husband) who stayed at home during the study period was included for the interview.

\section{Data collection and analysis methods}

Data regarding the socio-demographic characteristics, causes of mental illness and treatment preferences were collected using semi -structured questionnaire, through face to face interview by trained data collectors. The data was checked for its completeness and entered in to excel, and then it was exported to SPSS version 20 for data analysis. The results of the study were expressed quantitatively. The study results were presented using descriptive statistics like frequency, percentage, mean, standard deviation and inferential statistics Chi square testat significance level of $\mathrm{p}<0.05$.

\section{Results}

\section{Socio-demographic characteristics of respondents}

The households were selected from the two villages by systematic simple random sampling. A total of 141 participants from the two villages [Embaderho $(n=73)$ and Geshinashim $(n=68)$ ] were selected. Out of the total respondents $120(85.1 \%)$ and $21(14.9 \%)$ were females and males, respectively. Mean age of participants was 41.36 years $(\mathrm{SD} \pm 14.32)$. Majority of the study participants were orthodox $(93.6 \%)$ by religion and from Tigrigna ethnic group (99.3\%). Seventy three $(51.8 \%)$ of the study participants were below junior high school education and majority $(80.1 \%)$ were married. Seventy eight $(55.3 \%)$ and thirty two $(22.7 \%)$ of the respondents were housewife and farmers, and the majority (85.8\%) had an income of less than1, 500 Eritrean Nakfa (Table 1).

Table 1 Socio-demographic characteristics of respondents $(n=141)$

\begin{tabular}{|c|c|c|}
\hline Variable & Frequency (N) & Percentage $(\%)$ \\
\hline \multicolumn{3}{|l|}{ Age } \\
\hline 18- 19 & 2 & 1.4 \\
\hline $20-39$ & 71 & 50.4 \\
\hline $40-59$ & 50 & 35.5 \\
\hline $60-79$ & 14 & 9.9 \\
\hline$\geq 80$ & 4 & 2.8 \\
\hline \multicolumn{3}{|l|}{ Sex } \\
\hline Male & 21 & 14.9 \\
\hline Female & 120 & 85.1 \\
\hline \multicolumn{3}{|l|}{ Religion } \\
\hline Orthodox & 132 & 93.6 \\
\hline Catholic & 2 & 1.4 \\
\hline Protestant & 7 & 5 \\
\hline \multicolumn{3}{|l|}{ Ethnicity } \\
\hline Tigrigna & 140 & 99.3 \\
\hline Tigre & 1 & 0.7 \\
\hline \multicolumn{3}{|l|}{ Educational status } \\
\hline Cannot read and write & 23 & 16.3 \\
\hline Can read and write & 16 & 11.3 \\
\hline
\end{tabular}


Table Continued

\begin{tabular}{|c|c|c|}
\hline Variable & Frequency $(\mathbf{N})$ & Percentage (\%) \\
\hline Primary School & 34 & 24.1 \\
\hline Junior School & 31 & 22 \\
\hline Secondary School & 32 & 22.7 \\
\hline College and above & 5 & 3.5 \\
\hline \multicolumn{3}{|l|}{ Marital status } \\
\hline Single & 5 & 3.5 \\
\hline Married & 113 & 80.1 \\
\hline Living together & 2 & 1.4 \\
\hline Widowed & 15 & 10.6 \\
\hline Divorced & 4 & 2.8 \\
\hline Separated & 2 & 1.4 \\
\hline \multicolumn{3}{|l|}{ Occupational status } \\
\hline House wife & 78 & 55.3 \\
\hline Farmer & 32 & 22.7 \\
\hline Civil servant & 4 & 2.8 \\
\hline Military & 8 & 5.7 \\
\hline Government employee & 4 & 2.8 \\
\hline Trader & 3 & 2.1 \\
\hline Self employed & 5 & 3.5 \\
\hline Unemployed & 7 & 5 \\
\hline \multicolumn{3}{|c|}{ Family income in Eritrean Nakfa } \\
\hline$<500$ & 42 & 29.8 \\
\hline $500-999$ & 62 & 44 \\
\hline $1000-1499$ & 17 & 12.1 \\
\hline $1500-1999$ & 12 & 8.5 \\
\hline$\geq 2000$ & 8 & 5.7 \\
\hline Total & 141 & 100 \\
\hline
\end{tabular}

\section{Community knowledge and perception towards mental illness}

All study participants (141) were asked nine questions about their perception towards mental illness. They were asked whether they strongly Disagree, Disagree, Not Sure, Agree or Strongly Agree. The study participants were questioned their response whether substance misuse, head injury, communicable and non-communicable diseases, stress in daily life, punishment from God and Evil sprite can cause mental illnesses or it can be genetically inherited. Majority (70.9\%) of the respondents either agreed (45.4\%), or strongly agreed (25.5\%), whereas a total $20.6 \%$ strongly disagreed $(5.7 \%)$ and disagreed $(14.9 \%)$, that substance misuse can cause mental illnesses. Most of the respondents a total of $40.4 \%$ strongly disagree $(14.2 \%)$ or disagreed $(26.2 \%)$, but $33.3 \%$ and $8.5 \%$ were agreed and strongly agreed that mental illness can be genetically inherited. A total of (83.4\%) of the respondents either agreed $(45.8 \%)$ or strongly agreed $(37.6 \%)$ that mental illness can develop following head injury (Table 2).

They were asked that whether mental illness is treatable or infectious and the same results were documented as strongly disagree and disagree $(31.9 \%)$, not sure $(31.2 \%)$ and, agree and strongly agree $(36.9 \%)$, that communicable and non-communicable diseases (STDs, HIV/AIDS, hypertension, diabetes etc...) can cause mental illness or not. A total of $70.9 \%$ agreed ( $54.6 \%$ agreed and $16.3 \%$ strongly agreed) that mental illness is treatable disease like any other illnesses and $83 \%$ agreed (52.5\% agreed and $30.5 \%$ strongly agreed) it can be caused by stress in daily life; whereas $87.2 \%$ (61\% strongly disagreed and $26.2 \%$ disagreed) in indicating that it is not contagious or infectious. Out of these 141 study participants a total of $64.5 \%$ disagreed $(31.2 \%$ strongly disagreed and $33.3 \%$ disagreed) that mental illness is caused due to punishment from God, and $49.7 \%$ disagreed $(21.3 \%$ strongly disagreed and $28.4 \%$ disagreed) that mental illness is caused by Evil sprites (Table 2).

Table 2 Community knowledge and perception towards mental illness ( $\mathrm{n}=141)$

\begin{tabular}{|c|c|c|c|c|c|c|c|c|c|c|c|}
\hline \multirow{2}{*}{ No } & \multirow{2}{*}{ Question } & \multicolumn{2}{|l|}{1} & \multicolumn{2}{|l|}{2} & \multicolumn{2}{|l|}{3} & \multicolumn{2}{|l|}{4} & \multicolumn{2}{|l|}{5} \\
\hline & & $\mathbf{N}$ & $\%$ & $\mathbf{N}$ & $\%$ & $\mathbf{N}$ & $\%$ & $\mathbf{N}$ & $\%$ & $\mathbf{N}$ & $\%$ \\
\hline 1 & $\begin{array}{l}\text { Substance misuse like alcohol or drug can cause mental } \\
\text { illness }\end{array}$ & 8 & 5.7 & 21 & 14.9 & 12 & 8.5 & 64 & 45.4 & 36 & 26 \\
\hline 2 & Mental illness can be genetically inherited & 20 & 14 & 37 & 26.2 & 25 & 17.7 & 47 & 33.3 & 12 & 8.5 \\
\hline 3 & Mental illness can develop as a result head injury. & 7 & 5 & 8 & 5.7 & 7 & 5 & 66 & 45.8 & 53 & 38 \\
\hline 4 & $\begin{array}{l}\text { Communicable and non-communicable diseases (STDs, } \\
\text { HIV/AIDS, hypertension, diabetes) can cause mental illness }\end{array}$ & 13 & 9.2 & 32 & 22.7 & 44 & 31.2 & 43 & 30.5 & 9 & 6.4 \\
\hline 5 & Mental illness is treatable & 6 & 4.3 & 16 & 11.3 & 19 & 13.5 & 77 & 54.6 & 23 & 16 \\
\hline 6 & Stress in daily life can cause mental Illness & 2 & 1.4 & 13 & 9.2 & 9 & 6.4 & 74 & 52.5 & 43 & 31 \\
\hline 7 & Mental illness is infectious & 86 & 61 & 37 & 26.2 & 14 & 9.9 & 2 & 1.4 & 2 & 1.4 \\
\hline 8 & Mental illness is punishment from God & 44 & 31 & 47 & 33.3 & 14 & 9.9 & 22 & 15.6 & 14 & 9.9 \\
\hline 9 & Mental illness can be caused by Evil sprite & 30 & 21 & 40 & 28.4 & 25 & 17.7 & 36 & 25.5 & 10 & 7.1 \\
\hline
\end{tabular}

1= Strongly Disagree; 2 = Disagree; $3=$ Not Sure; 4 =Agree; 5 = Strongly Agree 


\section{Preferred place of treatment mental illness}

Majority $(85.1 \%)$ of the respondents reported that mental illness is a curable disease and $68.8 \%$ indicated that their best preferable site for treatment Hospital or Health Center in order to find modern medical treatment, where as the remaining $31.2 \%$ had preferred to go to traditional or spiritual healers (Table 3).

Table 3 Preferred place of treatment mental illness $(n=141)$

\begin{tabular}{lcl}
\hline Variable & Frequency $(\mathbf{N})$ & Percentage $\mathbf{( \% )}$ \\
\hline \multicolumn{2}{l}{ Mental illness curable like any other illness } & \\
Yes & 120 & 85.1 \\
No & 21 & 14.9 \\
Preferred place of treatment for Mental illness & \\
Hospital/Health Center & 97 & 68.8 \\
Traditional / Spiritual & 44 & 31.2 \\
Total & 141 & 100 \\
\hline
\end{tabular}

\section{Association between age and preferred place of treatment for mental illness}

Preference to treatment for mental illness to traditional or spiritual healers was observed to increase as age of the participants increases; whereas the study results also revealed that high preference of modern medical treatment among the literate than the illiterate participants. The study results showed no significant association between sex $(p$-value $=0.350)$, monthly income $(p$-value $=0.164)$ but a statistically significant association was found between preferred place of treatment mental illness, age $(p$-value $=0.032)$ and literacy $(p$-value $=0.000)$ (Table 4).

Table 4 Association between age and educational status, and preferred place of treatment for mental illness $(\mathrm{n}=141)$

\begin{tabular}{|c|c|c|c|}
\hline \multirow{2}{*}{ Variable } & \multicolumn{2}{|c|}{$\begin{array}{l}\text { Preferred place of treatment for mental } \\
\text { illness }\end{array}$} & \multirow[t]{2}{*}{$P$ - value } \\
\hline & $\begin{array}{l}\text { Hospital / Health } \\
\text { Center }\end{array}$ & Traditional / Spiritual & \\
\hline \multicolumn{4}{|l|}{ Age } \\
\hline$<40$ & $57(78.1 \%)$ & $16(21.9 \%)$ & \\
\hline $40-59$ & $32(64 \%)$ & $18(36 \%)$ & \multirow{2}{*}{0.032} \\
\hline $60-79$ & $7(50 \%)$ & $7(50 \%)$ & \\
\hline$\geq 80$ & $1(25 \%)$ & $3(75 \%)$ & \\
\hline Total & $97(68.8)$ & $44(31.2 \%)$ & $141(100)$ \\
\hline \multicolumn{4}{|l|}{ Sex } \\
\hline Male & $16(76.2 \%)$ & $5(23.8 \%)$ & \multirow{2}{*}{0.35} \\
\hline Female & $79(65.8 \%)$ & $41(34.2 \%)$ & \\
\hline Total & $95(67.4 \%)$ & $46(42.6 \%)$ & $141(100)$ \\
\hline \multicolumn{4}{|c|}{ Educational Status } \\
\hline Illiterate & $8(34.8 \%)$ & $15(65.2 \%)$ & \multirow{2}{*}{0.000} \\
\hline literate & $87(73.7 \%)$ & $31(26.3 \%)$ & \\
\hline Total & $95(67.4 \%)$ & $46(32.6 \%)$ & $141(100)$ \\
\hline \multicolumn{4}{|c|}{ Family income in Eritrean Nakfa } \\
\hline$<1000$ & $71(68.3)$ & $33(31.7 \%)$ & \multirow{3}{*}{0.164} \\
\hline 1000- 1999 & $21(72.4)$ & $8(27.6 \%)$ & \\
\hline$\geq 2000$ & $3(37.5 \%)$ & $5(62.5 \%)$ & \\
\hline Total & $95(67.4 \%)$ & $46(32.6 \%)$ & $141(100)$ \\
\hline
\end{tabular}

\section{Discussion}

Out of the total participants $(\mathrm{N}=141)$ from the two villages [Embaderho $(\mathrm{n}=73)$ and Geshinashim $(\mathrm{n}=68)], 120 \quad(85.1 \%)$ were females. Mean age of the study participants was 41.36 years $(\mathrm{SD} \pm 14.32)$. The study participants $(\mathrm{n}=141)$ were asked nine questions about their perception towards mental illness. They were asked whether they strongly disagree, disagree, not sure, agree or strongly agree. In Eritrea even though drug and substance abuse are highly uncommon, majority (70.9\%) of the respondents either agreed or strongly agreed that mental illness can be caused by substance, drug and alcohol abuse. The results of this study were found to be in line to studies on mental illness stigma and discrimination in Zambia, Tanzania and Australia, that documented community perception and knowledge on the causes of mental illnesses are caused by evil spirits; substance, drug and alcohol abuse. ${ }^{7-9}$ A study on co-morbidity of substance misuse and mental illness in a community also reported a higher prevalence of psychiatric disorders among alcohol addicts. ${ }^{10-12}$

The study results also showed $40.4 \%$ of the respondents either strongly disagreed $(14.2 \%)$ or disagreed $(26.2 \%)$, but $33.3 \%$ and $8.5 \%$ were agreed and strongly agreed that mental illness is genetically inherited disease, this indicates poor knowledge and perception on its inheritance among the study participants. A total of $83.4 \%$ of the respondents either agreed $(45.8 \%)$ or strongly agreed $(37.6 \%)$ that mental illness can develop following head injury. The same results were found whether communicable and non-communicable diseases (STDs, HIV/AIDS, hypertension, diabetes etc...) cause mental illness or not, as strongly disagree and disagree $(31.9 \%)$, not sure $(31.2 \%)$ and, agree and strongly agree $(36.9 \%)$. Majority of the respondents $70.9 \%$ agreed $(54.6 \%$ agreed and $16.3 \%$ strongly agreed) that mental illness is treatable disease like any other illnesses and $83 \%$ agreed $(52.5 \%$ agreed and $30.5 \%$ strongly agreed), that it can be caused by stress in daily life. This study has consistency with the reports of National Center for Chronic Disease Prevention and Health Promotion Division of Population Health that documented; both chronic communicable and non-communicable diseases including mental illnesses are disabling diseases regardless of age, culture, race or ethnicity, gender, or income. The report also elaborated a significant relationship between mental health, chronic disease and injury. ${ }^{13}$ Majority (87.2\%) of the participant opposed for the transmitability of mental illness as a contagious or infectious disease.

A total of $64.5 \%$ either disagreed or strongly disagree that mental illness is neither caused by punishment from God or Evil sprites. These differences of causation in different countries are usually related to culture, ethnicity, religion, educational status and others. Therefore, the findings of this study were found to have some inconsistencies with other studies as mental health perception, knowledge and preference of treatment in different communities' can be affected multi-dimensionally. A cross sectional study on "Factors Influencing Adolescents Stigmatizing Attitudes and Perception of Community Reaction towards Mental Illness" that was conducted in Nigeria indicated that majority $(72.8 \%)$ of the respondents had knowledge on the causes of mental illness and $76.1 \%$ were able to describe that it is not a punishment from God, a result of stress or bad coping mechanism to difficult situation. ${ }^{14}$ Despite the advancement in modern medicine still there are people who perceive mental illness as a disease that can be due to the hatred of witchcraft or evil-eyed. There are studies that reported mental illness as a disease that can be witched and needs a combination of spiritual interventions and medical therapy for its treatment. ${ }^{6,15}$ There are studies that documented mental illness as a 
disease caused by supernatural powers and treated by traditional or spiritual healers. A study that was conducted in Ethiopia reported that supernatural powers as cause of mental illness, traditional healing and healers as preferable treatment method. ${ }^{16}$

Majority $(85.1 \%)$ of the study participants responded that mental illness as a curable disease and $68.8 \%$ acknowledged that modern medical approach mainly at Hospital or Health Center as their best preferable site of treatment, whereas the remaining $31.2 \%$ replied that their best preference as to go for Traditional or Spiritual healers. This results may be due to increased awareness, level of education and geographic access to the capital and healthy facilities. There are people or communities that perceive mental illness as a disease that is caused by people, evil sprites, by eating or something that is polluted by someone knowingly etc.... If the case is that they consider it can be cured only by traditional or spiritual healer rather than the modern medical approach. A qualitative study on the management of perceived mental health problems by spiritual healers in Nigeria, indicated that most of the mentally ill people are treated by traditional or spiritual healers that include; holy water, patient beating for driving away the evil, praying in the church. ${ }^{17}$ Preference on treatment for mental illness to traditional or spiritual healers was observed to decrease as the age of participants increases. The study results showed no significant association between sex, monthly income and preference of modern medical approach, but high preference of modern medical treatment among the younger age group and literate participants was found to be statistically significant at $p$-value $=0.032$ and $p$-value $=0.000$. This study has consistency with other studies that were conducted in Nepal and Tanzania that identified the best preference of treatment as the modern medical approach. ${ }^{8,18}$

\section{Conclusion}

The study results revealed poor knowledge and perception of mental illnesses among who cannot read and write (illiterate) and old aged people. High preference to modern medical approach was identified with significant association to literacy and young age group. Therefore, the study results recommend an intensified health promotion activity to create awareness and clear understanding that mental illness is not caused by evil spirits, punishment from God or bewitched and to make them understand and follow the correct way and site of treatment approach which is modern medical approach. Such a strategy can be implemented by utilizing community health workers in an area with a shortage of mental health care staff.

\section{Acknowledgements}

The authors are grateful for Dr. Haile Mihtsun for his endorsement for this research and endless encouragement and help towards conducting research. We would also like to thank for the heads of the respective health facilities and village administrators. Finally, we would like to thank for the study participants who provided us the required information.

\section{Conflict of interest}

The author declares that there is no conflict of interest.

\section{References}

1. World Health Organization. A conceptual framework for action on the social determinants of health. 2010.
2. Monteiro NM. Addressing mental illness in Africa: Global health challenges and local opportunities. Community Psychology in Global Perspective. 2015;10:1(2):78-95.

3. Schoonover J, Lipkin S, Javid M, et al. Perceptions of traditional healing for mental illness in rural Gujarat. Annals of global health. 2014;80(2):96-102.

4. Benti M, Ebrahim J, Awoke T, et al. Community Perception towards Mental Illness among Residents of Gimbi Town, Western Ethiopia. Psychiatry journal. 2016;2016:8

5. James MM. Stigma towards mental illness and the mentally ill in Kenya. 2007.

6. Kinyua B, Njagi E. Knowledge and perceptions about mental illnesses among kenyan immigrants living in jyvaskyla, finland. 2013.

7. Kapungwe A, Cooper S, Mwanza J, et al. Mental illness-stigma and discrimination in Zambia. African Journal of Psychiatry. 2010;13(3):192-203.

8. Benedicto M, Mndeme E, Mwakagile D, et al. Community Knowledge, Attitudes and Perception towards Mental Illness in Dodoma Municipality, Tanzania. ARC Journal of Public Health and Community Medicine. 2016;1(3):10-18.

9. Denton M, Foster M, Bland R. How the prison-to-community transition risk environment influences the experience of men with co-occurring mental health and substance use disorder. Australian \& New Zealand Journal of Criminology. 2017;50(1):39-55.

10. Weaver T, Madden P, Charles V, et al. Comorbidity of substance misuse and mental illness in community mental health and substance misuse services. The British Journal of Psychiatry. 2003;183(4):304-313.

11. Morisano D, Babor TF, Robaina KA. Co-occurrence of substance use disorders with other psychiatric disorders: Implications for treatment services. Nordic studies on alcohol and drugs. 2014;31(1):5-25.

12. Indig D, Gear C, Wilhelm K. Comorbid substance use disorders and mental health disorders among New Zealand prisoners. New Zealand Department of Corrections. 2016.

13. Centers for Disease Control and Prevention, Substance Abuse and Mental Health Services Administration, National Association of County Behavioral Health \& Developmental Disability Directors, National Institute of Mental Health, The Carter Center Mental Health Program. Attitudes Toward Mental Illness: Results from the Behavioral Risk Factor Surveillance System. Atlanta (GA); Centers for Disease Control and Prevention; 2012.

14. Akinbode O, Tolulope. Factors Influencing Adolescents Stigmatising Attitudes and Perception of Community Reaction towards Mental Illness in Nigeria. Sudan Journal of Medical Sciences (SJMS). 2017;12(4):240-252.

15. Sokhela D. Mental Illness in the Context of Witchcraft and Bewitching. A South African Perspective: Voices from Communities. Mental Health in Family Medicine. 2016;12:299-300.

16. Tibebe A, Tesfay K. Public Knowledge and Beliefs about Mental Disorders in Developing Countries: A Review. Journal of Depression Anxiety S3 (004). 2015;2167-1044.

17. Agara AJ, Makanjuola AB, Morakinyo OB. Management of perceived mental health problems by spiritual. African Journal of Psychiatry. 2008;11(2):113-118.

18. Lama S. Attitudes and Perceptions of Mental Disorders among Individuals from Nepal. 2013. 